\title{
Paul Vidal de la Blache (1845-1918)
}

\author{
"DES CARACTÈRES \\ DISTINCTIFS DE LA \\ GÉOGRAPHIE"
}

\section{3}

Un document produit en version numérique par Michel Côté, bénévole, Étudiant en géographie à l'Université Laval de Québec

Courriel: cote_mic@hotmail.com

Dans le cadre de la collection: "Les classiques des sciences sociales" dirigée et fondée par Jean-Marie Tremblay, professeur de sociologie au Cégep de Chicoutimi

Site web: http://www.uqac.uquebec.ca/zone30/Classiques_des_sciences_sociales/index.html

Une collection développée en collaboration avec la Bibliothèque

Paul-Émile-Boulet de l'Université du Québec à Chicoutimi

Site web: http://bibliotheque.uqac.uquebec.ca/index.htm 
Un document produit en version numérique par M. Michel Côté, bénévole, Étudiant en géographie à l'Université Laval de Québec

Courriel: cote_mic@hotmail.com à partir de :

\section{Paul Vidal de la Blache (1745-1918) \\ "Des caractères distinctifs de la géographie"}

Une édition électronique réalisée à partir de l'article de Paul Vidal de la Blache, "Des caractères distinctifs de la géographie" in revue Annales de Géographie, tome 22, no 124, pages 289 à 299. Paris : Armand Colin, Éditeur.

Polices de caractères utilisée :

Pour le texte: Times New Roman, 12 points.

Pour les notes de bas de page : Times New Roman, 10 points.

Édition électronique réalisée avec le traitement de textes Microsoft Word 2001.

Mise en page sur papier format

LETTRE (US letter), 8.5' x 11'”)

\section{Fait avec}

Macintosh

Édition complétée le 8 avril 2003 à Chicoutimi, Québec. 


\section{Table des matières}

I- l'unité terrestre.

II- la combinaison des phénomènes.

III- les surfaces.

IV- la force du milieu et l'adaptation.

V- la méthode descriptive.

VI- la géographie et l'histoire. 
Appelé à parler de géographie devant un auditoire de futurs maîtres formés aux méthodes scientifiques, mais se préparant à des enseignements divers, je me suis demandé, non sans embarras, quelle était, parmi les questions que soulève un tel sujet, celle qui convenait le mieux en la circonstance. J'ai été frappé, à la réflexion, des malentendus qui règnent sur l'idée même de géographie. Dans le groupe des sciences naturelles auxquelles elle se rattache sans nul doute, elle tient une place à part. Ses affinités n'excluent pas de sensibles différences. Or, c'est surtout sur ces différences que les idées manquent de précision. Il m'a semblé qu'en essayant de porter quelque lumière sur ce côté des choses, c'est-à-dire en m'attachant à spécifier ce qui distingue la géographie, je me conformerai à l'intention qui préside à ces conférences. La pédagogie est une oeuvre de coordination et de rapports ; ne doit-elle pas être considérée comme une sorte de philosophie embrassant dans une vue d'ensemble ce qui contribue à la formation de l'esprit ? La géographie est tenue de puiser aux mêmes sources de faits que la géologie, la physique, les sciences naturelles et, à certains égards, les sciences sociologiques. Elle se sert de notions dont quelques-unes sont l'objet d'études approfondies dans des sciences voisines. De là vient, pour le dire en passant, le reproche qui lui est parfois adressé de vivre d'emprunts, d'intervenir indiscrètement dans le champ d'autrui, comme s'il y avait des compartiments réservés dans le domaine de la science. Gardons-nous d'attacher à ces critiques plus d'importance que ne leur en attribuent sans doute leurs auteurs. En réalité, comme nous verrons, la géographie a bien un domaine qui lui est propre. L'essentiel est de considérer quel usage elle fait des données sur lesquelles elle s'exerce. Leur applique-telle des méthodes qui lui appartiennent? Apporte-t-elle des points de vue d'où les choses puissent apparaître en perspective spéciale, qui les montre sous un jour nouveau ? Toute la question est là. Dans la complexité des phénomènes qui s'entre-croisent dans la nature, il ne doit pas y avoir une seule manière d'aborder l'étude des faits; il est utile qu'ils soient envisagés sous des angles différents. Et si la géographie reprend à son compte certaines données qui portent une autre estampille, il n'y a rien dans cette appropriation qu'on puisse taxer d'anti-scientifique. 


\section{I- l'unité terrestre.}

\section{$\underline{\text { Retour à la table des matières }}$}

La géographie comprend par définition l'ensemble de la terre. Ce fut le mérite des mathématiciens-géographes de l'antiquité, Érathosthènes, Hipparque, Ptolémée, de poser en principe l'unité terrestre, de faire prévaloir cette notion au-dessus des descriptions empiriques de contrées. C'est sur cette base que la géographie a pu se développer comme science. L'idée de correspondance, de solidarité entre les phénomènes terrestres, a pénétré ainsi et pris corps, fort lentement il est vrai, car il s'agissait de l'appuyer sur des faits, et non sur de simples hypothèses. Lorsque, au commencement du XIXe siècle, Alexandre De Humboldt et Carl Ritter se firent les initiateurs de ce qu'on appelait alors la géographie comparée, ils se guidaient d'après une vue générale du globe ; et c'est à ce titre que leur impulsion fut féconde. Tous les progrès accomplis depuis dans la connaissance de la terre se sont accordés à mieux mettre en lumière ce principe d'unité. S'il est un domaine où il se manifeste avec une souveraine clarté, c'est celui des masses liquides qui couvrent les trois quarts du globe et de l'océan atmosphérique qui l'enveloppe. Dans les mouvements de l'atmosphère, écrit le météorologiste Dove, "aucune partie ne peut s'isoler, chacune agit sur sa voisine " .C'est ainsi qu'en se répercutant, les bourrasques formées aux abords de Terre-Neuve abordent les côtes de l'Europe occidentale et par contre-coup le nord de la Méditerranée ; et si on les perd de vue ensuite et que leur marche échappe aux observatoires, il n'est pas douteux que la série des répercussions se poursuive. Les parties de l'océan sont mises en communication intime par une circulation de fonds et de surface. " Quum Oceanus Movetur, Totus Movetur ", écrivait déjà Bernard Varenius.

La partie solide du globe ne subit pas moins l'action d'une dynamique générale. L'ensemble des faits tectoniques que les explorations poussées dans les diverses contrées de la terre ont mis en lumière, montre assez de coordination pour qu'Édouard Suess ait pu édifier sur eux une synthèse, dont l'idée même eût auparavant paru chimérique. La connaissance des régions polaires nous promet enfin de nouveaux exemples de correspondance et de corrélation, qui éclaireront sans doute d'un jour nouveau la genèse des phénomènes. Cette idée d'unité est commune sans doute à toutes les sciences qui touchent à la 
physique terrestre, de même qu'à celles qui étudient la répartition de la vie. L'insolation, l'évaporation, la chaleur spécifique de la terre et de l'eau, les changements d'état de la vapeur d'eau, etc., s'éclairent par la comparaison réciproque des diverses parties du globe. La loi de pesanteur domine toute la diversité des formes d'érosion et d'entraînement, et se manifeste ainsi dans sa plénitude. Toute espèce vivante est dans une perpétuelle tension d'efforts pour acquérir ou défendre un espace qui lui permette de subsister, et cela sert de guide au naturaliste. La connaissance de ces faits qui, dans des ordres divers et à des degrés différents, contribuent à fixer la physionomie de la terre, résulte d'un ensemble d'observations où chaque partie du globe doit, autant que possible, apporter son témoignage. Chaque science accomplit en ce sens la tâche qui lui est propre; mais on ne peut pas dire qu'elle remplisse pour cela le rôle de la géographie : c'est ce rôle donc qu'il s'agit de préciser.

\section{II- la combinaison des phénomènes.}

$\underline{\text { Retour à la table des matières }}$

Je ne saurais mieux le faire qu'en empruntant à l'auteur d'un des meilleurs ouvrages qui aient été publiés sur la climatologie, le professeur J Hann, les termes dont il se sert pour établir la distinction entre la météorologie et l'étude des climats. " celle-ci, dit-il, est de sa nature plus descriptive ;elle a pour objet de livrer au lecteur une image aussi vivante que possible de l'action combinée de tous les phénomènes atmosphériques sur une partie de la terre. " on peut dire, en généralisant cette remarque, que la géographie, s'inspirant comme les sciences voisines de l'idée d'unité terrestre, a pour mission spéciale de chercher comment les lois physiques ou biologiques qui régissent le globe, se combinent et se modifient en s'appliquant aux diverses parties de la surface. Elle les suit dans leurs combinaisons et leurs interférences. La terre lui fournit pour cela un champ presque inépuisable d'observations et d'expériences. Elle a pour charge spéciale d'étudier les expressions changeantes que revêt suivant les lieux la physionomie de la terre. Remarquons, avant d'aller plus loin, que cette combinaison est la forme même sous laquelle les phénomènes s'offrent partout dans la nature. La géographie est sollicitée vers les réalités. " dans la nature, a dit Buffon, la plupart des effets dépendent de plusieurs causes différemment combinées. " avec plus de précision encore, le penseur éminent, très attentif aux choses géographiques, que fut Henri Poincaré, s'exprime ainsi 
dans un de ses derniers écrits : " l'état du monde, et même d'une très petite partie du monde, est quelque chose d'extrêmement complexe et qui dépend d'un très grand nombre d'éléments. "la justesse de ces vues nous frappe, quelle que soit la partie de la géographie que nous considérions. Le modelé du sol résulte du conflit entre les énergies que déploient pour l'attaque les agents météoriques et la force de résistance que leur opposent les roches ; mais ce conflit s'exerce sur un champ qui a déjà été remanié dans le cours des âges, et qui l'est encore incessamment suivant les modifications des niveaux de base et les oscillations de climat. Ce qu'on appelle le climat d'une contrée est une moyenne à laquelle contribuent la température, l'humidité, la luminosité, les vents ; mais l'évaluation de ces divers éléments ne donnerait qu'une idée fort incomplète, si l'on ne cherchait pas de quelle façon ils se combinent, non seulement entre eux, mais avec le relief, l'orientation, les formes du sol, la végétation et même les cultures. Voit-on, par exemple, le maximum saisonnier de chaleur coïncider avec le maximum d'humidité ? Tous les caractères d'un certain type de climat, celui du sud de la Méditerranée, se dressent devant l'esprit. D'autres types, avec de multiples nuances, correspondent, au contraire, aux divers régimes de pluies d'été. La diversité d'éléments à considérer n'est pas moindre dans le domaine des êtres vivants. La végétation d'une contrée est un ensemble composite, dans lequel on distingue des plantes de provenances diverses : les unes envahissantes, les autres réfugiées, d'autres qui sont des legs de climats antérieurs, d'autres qui ont suivi d'elles-mêmes les cultures de l'homme. Tout indique aussi, à mesure que l'on avance dans l'examen et l'analyse des faunes régionales, leur caractère composite. Des migrations, dont le sens et les dates nous échappent le plus souvent, ont brassé les tribus d'êtres vivants, y compris les hommes ; et c'est de leurs résidus que se sont formés, sur les diverses contrées où ils ont pu se concentrer, les occupants qu'on y rencontre. Tandis que les classifications linguistiques nous donnent l'illusion de grands groupes humains, les indices que fournissent l'anthropologie et la préhistoire s'accordent à montrer la diversité des races qui, à la manière d'alluvions successives, ont formé la plupart de nos peuplements. L'analyse de ces éléments, l'étude de leurs rapports et de leurs combinaisons, composent la trame de toute recherche géographique. Il ne peut plus être question, d'après ce point de vue, d'une antinomie de principe entre deux sortes de géographie : l'une qui, sous le nom de géographie générale, en serait la partie vraiment scientifique ; et l'autre qui s'appliquerait, sans autre fil conducteur qu'une curiosité superficielle, à la description des contrées. De quelque côté qu'on les envisage, ce sont les mêmes faits généraux, dans leurs enchaînements et leur corrélation, qui s'imposent à l'attention. Ces causes, s'il est permis de se servir de ce mot ambitieux, engendrent en se combinant les variétés sur lesquelles le géographe travaille : soit qu'il se propose de déterminer des types de climats, de formes de sol, d'habitat, etc., comme il le fait quand il traite de géographie 
générale ; soit qu'il s'efforce de caractériser des contrées, de les peindre même, car le pittoresque ne lui est pas interdit.

\section{III- les surfaces.}

$\underline{\text { Retour à la table des matières }}$

Le champ d'étude par excellence de la géographie, c'est la surface ; c'est-àdire l'ensemble des phénomènes qui se produisent dans la zone de contact entre les masses solides, liquides et gazeuses qui constituent la planète. Ce contact est le principe de phénomènes sans nombre, dont quelques-uns à peine soupçonnés encore ; il agit comme un réactif pour mettre en évidence les énergies terrestres. La colonne d'air se modifie sans cesse au contact des surfaces solides ou liquides ; et la vapeur d'eau, transportée à la suite de ces oscillations, s'accroît, se condense ou se précipite suivant l'état thermique des surfaces qu'elle rencontre. Le sol est en butte de la part des météores, non seulement à des attaques de vive force, mais à des atteintes par infiltration. Son épiderme se durcit, ou bien il se décompose à leur contact. L'air et l'eau pénètrent alors dans sa texture ameublie ; et la terre devient, suivant l'expression de Berthelot, quelque chose de vivant. Les ferments et les bactéries entrent en mouvement ; l'acide carbonique dissout les phosphates, la chaux, la potasse et autres ingrédients qui entrent dans le corps des plantes, et qui s'y élaborent, sous l'action de la lumière, pour servir de nourriture aux autres êtres vivants. Sans doute l'intérieur de la terre est le siège d'autres phénomènes de transformation, d'incalculable portée.

La géographie toutefois n'y est qu'indirectement intéressée. S'il est à peu près certain que les plissements et renversements qui prennent un aspect si saisissant dans certaines chaînes de montagnes, se sont formés en profondeur sous l'effort de pressions et de contractions énormes, cette oeuvre souterraine ne devient un objet géographique que lorsque par l'action combinée des soulèvements et des dénudations elle apparaît à la surface. Elle prend alors place dans le relief, s'associe aux autres formes du sol, influe sur le modelé de ce qui l'entoure ; et elle devient un des plus puissants centres d'action sur le climat, l'hydrographie, la végétation et les hommes. Parmi les surfaces qu'étu-

die la géographie, celles de la lithosphère ont l'avantage de conserver plus ou moins l'empreinte des modifications qu'elles ont éprouvées depuis leur 
émersion. Elles présentent par là un intérêt particulier et ouvrent une nouvelle source d'enseignements. C'est comme un tableau enregistreur, sur lequel l'état présent des formes se montre la continuation d'états antérieurs. à travers les formes qui appartiennent au cycle présent d'évolution, on discerne des linéaments de celles qui les ont précédées. Ils subsistent assez nets souvent pour qu'on distingue jusqu'à quel degré d'évolution étaient parvenues les formes du sol, dues à des actions de même nature que celles qui travaillent sous nos yeux, quand un nouveau cycle d'érosion s'est ouvert. Dans la chaîne des âges, c'est naturellement l'anneau le plus proche, l'antécédent immédiat qui a le moins souffert de l'usure. Il se transforme plutôt qu'il n'est aboli. L'œuvre du passé persiste à travers le présent comme la matière sur laquelle s'exercent les forces actuelles. Nous sommes dès lors en pleine géographie. Dans les contrées qu'avaient envahies les glaciers quaternaires, les cours d'eau n'ont pas fini de déblayer les débris qu'ils avaient accumulés. Quelques-uns cherchent encore leur lit à travers ces matériaux, dont ils forment des alluvions. Les vallées dont un climat plus humide avait sillonné le Sahara sont, en apparence du moins, des formes fossiles : elles exercent néanmoins une influence sensible sur les sources, les puits, la végétation, et le vent s'emparant de leurs alluvions sableuses, y trouve les matériaux des dunes qu'il édifie. L'aspect de la surface solide se décèle ainsi comme le résultat de modifications sans cesse remaniées d'âge en âge ; il représente une suite, et non un état une fois donné et atteint d'emblée. Les formes actuelles ne sont intelligibles que si on les envisage dans la succession dont elles font partie. Comment expliquer par exemple, sans recourir à la considération d'un régime de pentes antérieur, la direction si paradoxale en apparence de ces fleuves qui traversent, au lieu de les contourner, les obstacles qui semblent s'opposer à leur passage? Tout cela restait une énigme, tant que n'avait pas pénétré dans la science, à l'aide de la comparaison et de l'analyse, cette notion de l'évolution des formes qui en est la clef. On peut dire qu'elle domine aujourd'hui toute recherche.

\section{IV- la force du milieu et l'adaptation.}

\section{$\underline{\text { Retour à la table des matières }}$}

Si l'on vient à se placer dans l'état d'esprit du géographe, on se voit donc aux prises avec des facteurs d'ordre divers, de provenance hétérogène, et formant entre eux des combinaisons multiples ; on sent que l'équilibre qui résulte 
de ces combinaisons n'a rien d'absolument stable, qu'il est à la merci de modifications auxquelles la multiplicité des facteurs ouvre une ample marge. On peut se demander où est un principe directeur qui permette d'édifier sur ce terrain en apparence mouvant des méthodes qui se tiennent, et de tenter des essais coordonnés de descriptions terrestres. Recourons encore à l'observation. Ce que l'observation et l'analyse rencontrent sur ces surfaces où s'impriment les phénomènes, ce ne sont pas des cas isolés, des traits incohérents, mais des groupes de formes obéissant à une action d'ensemble, liées par des affinités, et travaillant de concert à éliminer de la surface ce qui ne convient plus aux conditions actuelles. Là où les cours d'eau n'ont plus la force d'entrâner les débris de destruction des roches, l'aspect tout entier du modelé porte l'empreinte de cette impuissance: d'étroites berges terreuses encaissant les thalwegs, de grandes surfaces unies au-dessus desquelles émergent çà et là des sommets coniques, composent d'une diversité de traits qui néanmoins convergent, l'ensemble classique du paysage de région aride. Le contraste est complet avec le monde de formes qui peuplent la surface, quand l'œuvre d'un déblaiement avancé a modelé les flancs des vallées, mis à nu les versants des montagnes, disséqué et diversifié les plans. Là où les glaciers ont passé, subsiste, au moins provisoirement, cet ensemble chaotique de monticules et de lacs qu'on appelle le paysage morainique. Le nom d'appareil littoral caractérise une affluence de formes qui, variées en elles-mêmes, n'apparaissent guère l'une sans l'autre : ici des fiords surmontés de lacs intérieurs et prolongés vers la mer par cette bordure déchiquetée d'îles et d'écueils que les scandinaves ont appelée Skiargaard; ailleurs la rangée uniforme des lagunes, des barres fluviales et des cordons littoraux. Chacun de ces types se compose de formes en dépendance réciproque. Telle est aussi la physionomie de la végétation. Ce n'est pas l'olivier qui personnifie à lui seul la végétation méditerranéenne, pas plus qu'une hirondelle ne fait le printemps. Ce que cette expression de Méditerranée évoque, c'est une multitude de plantes dont les formes ont par leur variété excitée l'imitation artistique, mais qui coexistent néanmoins dans un ensemble que le langage populaire désigne sous les noms de maquis, garigues, ou autres. C'est une des associations caractéristiques que distingue la science botanique. Partout donc nous rencontrons des expressions collectives, les unes populaires, les autres scientifiques, correspondant à ces faits d'observation. Elles suffiraient à nous avertir qu'un lien commun existe entre les divers éléments dont nous avions reconnu la complexité. De quoi est formé ce lien? C'est par cette question que nous sommes amenés à la notion de milieu ; notion dont l'apparence vague tient à l'abus qu'on en fait, mais qui, pour peu qu'on la creuse, se montre pleine d'enseignements. C'est le climat, peut-on dire en un sens, qui décide de la prépondérance des formes de déblaiement ou de dénudation. Mais l'explication est trop sommaire, et ce mot ne rend pas un compte adéquat et complet des phénomènes. Nous voyons en effet 
que les formes elles-mêmes cherchent à s'organiser entre elles, à réaliser un certain équilibre. Ici à l'aide du vent, ailleurs à l'aide des eaux courantes, elles travaillent d'après un plan et pour une fin déterminée; peu importe d'ailleurs qu'elles l'atteignent ou qu'elles soient contrecarrées en route. Les dunes et les sables s'alignent suivant une géométrie ; ils accomplissent une oeuvre de nivellement. Chaque flèche s'allonge dans le sens de sa voisine, et tend à se raccorder avec celle qui lui fait face. Le ravin qui, né d'une rigole, écorche le flanc d'une montagne, soutire un faisceau de rigoles semblables; et quand de cet ensemble il s'est formé un cours d'eau, celui-ci travaille, de concert avec ses frères, à adapter son profil suivant un niveau de base commun. Si dans le monde des formes inanimées les traits se coordonnent, cette adaptation réciproque n'est pas moins sensible entre les êtres vivants, mais elle s'exerce différemment. Les plantes qui peuplent une contrée, les animaux auxquels ces plantes servent de nourriture, et jusqu'à un certain point même les groupes humains qui trouvent dans cet entourage, cet " environnement ", suivant l'expression anglaise, le principe d'un genre de vie, sont composés d'éléments disparates. Il entre, avons-nous dit, dans les associations végétales les espèces les plus diverses de provenance et de forme. Mais au-dessus de ces différences une tonalité générale domine ; les plantes s'organisent physiologiquement, elles revêtent pour s'accommoder aux influences ambiantes une livrée commune, d'après l'altitude, les intempéries, la sécheresse, la chaleur humide. Non seulement elles modifient suivant des procédés divers et parfois très inattendus leurs organes extérieurs, mais elles se combinent entre elles de façon à se répartir l'espace. Dans ces groupements, qui sont l'aspect normal sous lequel se présente et se grave dans nos yeux la physionomie du paysage, chaque plante s'est arrangée avec ses voisines pour avoir sa part de sol, de lumière, de nourriture. Les êtres viennent s'associer et s'unir, "trouvant avantage et profit dans les conditions déterminées par la présence des autres". Une forêt est une sorte d'être collectif où coexistent, dans une harmonie provisoire et non à l'épreuve des changements, des arbres, des végétaux de sous-bois, des champignons et une foule d'hôtes également attitrés, insectes, termites, fourmis. Ainsi les choses se présentent à nous en groupes organisés, en associations régies par un équilibre que l'homme dérange incessamment ou, suivant les cas, redresse, en y portant la main. L'idée de milieu, dans ces expressions diverses, se précise comme corrélative et synonyme d'adaptation. Elle se manifeste par des séries de phénomènes qui s'enchainent entre eux et sont mis en mouvement par des causes générales. C'est par elle que nous sommes incessamment ramenés à ces causes de climat, de structure, de concurrence vitale, qui donnent le branle à une foule d'activités spéciales des formes et des êtres. 


\section{V- la méthode descriptive.}

$\underline{\text { Retour à la table des matières }}$

On peut juger, par ce qui vient d'être dit, quel rôle capital joue en tout ceci la description. La géographie se distingue comme science essentiellement descriptive. Non pas assurément qu'elle renonce à l'explication : l'étude des rapports des phénomènes, de leur enchaînement et de leur évolution, sont autant de chemins qui y mènent. Mais cet objet même l'oblige, plus que toute autre science, à suivre minutieusement la méthode descriptive. Une de ces tâches principales n'est-elle pas de localiser les divers ordres de faits qui la concernent, de déterminer exactement la position qu'ils occupent, l'aire qu'ils embrassent ? Aucun indice, aucune nuance même ne saurait passer inaperçue ; chacune a sa valeur géographique, soit comme dépendance, soit comme facteur, dans l'ensemble qu'il s'agit de rendre sensible. Il faut donc prendre sur le fait chacune des circonstances qui les caractérisent, et en dresser exactement le bilan. Dans le riche clavier de formes que la nature étale à nos yeux, les conditions sont si diverses, si entre-croisées, si complexes qu'elles risquent d'échapper à qui croit trop tôt les tenir. Deux écueils sont particulièrement à craindre : celui des formules trop simples et rigides entre lesquelles glissent les faits, et celui des formules à tel point multipliées qu'elles ajoutent à la nomenclature et non à la clarté. Décrire, définir et classer, pour de là déduire, sont des opérations qui logiquement se tiennent; mais les phénomènes naturels d'ordre géographique ne se plient pas avec un empressement toujours docile aux catégories de l'esprit. La description géographique doit être souple et variée comme son objet même. C'est souvent profit pour elle de puiser dans la terminologie populaire ; celle-ci s'étant formée directement en contact avec la nature, telle désignation saisie sur le vif, tel dicton rural ou proverbe peuvent ouvrir un jour sur un rapport, une périodicité, une coïncidence, toutes choses qui se réclament directement de la géographie. Ce n'est pas non plus sans raison que dans les livres ou mémoires géographiques les représentations figurées tiennent de plus en plus de place. Le dessin, la photographie entrent à titre de commentaires dans la description. Les figures schématiques ont leur utilité comme instrument de démonstration. Mais rien ne vaut le dessin comme moyen d'analyse pour serrer de près la réalité, et comme contrôle de ces observations directes, qui trouvent aujourd'hui dans les excursions géo- 
graphiques l'occasion fréquente de s'exercer. L'habitude de ces leçons itinérantes est, chez nous, un des plus remarquables gains pédagogiques de ces dernières années. C'est l'école de plein air, plus hygiénique et plus efficace que toute autre. Elle choisit d'avance ses textes, c'est-à-dire les paysages où se ramasse, dans une perspective plus facile à saisir, cet ensemble de traits caractéristiques qui gravent dans l'esprit du géographe l'idée de contrée.

\section{VI- la géographie et l'histoire.}

\section{$\underline{\text { Retour à la table des matières }}$}

Il va sans dire que dans cette physionomie l'homme, directement ou indirectement, par sa présence, par ses oeuvres ou par le contrecoup de ses oeuvres, s'impose toujours à l'attention. Lui aussi est un des agents puissants qui travaillent à modifier les surfaces. Il se range à ce titre parmi les facteurs géographiques de premier ordre. Son oeuvre sur la terre est déjà longue ; il est peu de parties qui n'en portent les stigmates. On peut dire que de lui dépend l'équilibre actuel du monde vivant. C'est une tout autre question que celle de savoir quelle influence les conditions géographiques ont exercée sur ses destinées et particulièrement sur son histoire. Je ne puis qu'effleurer ici ce point important. L'histoire et la géographie sont d'anciennes compagnes qui ont longtemps cheminé ensemble et qui, comme il arrive entre de vieilles connaissances, ont perdu l'habitude de discerner les différences qui les séparent. Loin de moi l'intention de troubler l'harmonie de ce ménage. Il est utile toutefois que, tout en continuant de se rendre de réciproques services, elles aient nettement conscience des divergences qui existent dans leur point de départ et leurs méthodes. La géographie est la science des lieux et non celle des hommes; elle s'intéresse aux événements de l'histoire en tant qu'ils mettent en oeuvre et en lumière, dans les contrées où ils se produisent, des propriétés, des virtualités qui sans eux seraient restées latentes. L'histoire d'Angleterre est insulaire, celle de la France est tiraillée entre la mer et le continent ; le doigt de la géographie est marqué sur chacune. Ces enchaînements historiques ont leur place dans l'évolution des faits terrestres; mais combien est limitée la période de temps qu'ils embrassent! C'est une sorte de 
truisme que d'opposer la brièveté de la vie humaine à la durée qu'exige la nature pour ses moindres changements : mais enfin, combien peu de générations suffirait-il de mettre bout à bout, pour toucher au terme au-delà duquel il n'y a plus de témoignage historique, et même, puisque l'histoire se résume en de grands efforts collectifs, où il n'y a plus d'histoire ! L'étude de l'évolution des phénomènes terrestres suppose l'emploi d'une chronologie qui diffère essentiellement de celle de l'histoire. On est trop porté à l'oublier. C'est ce qui arrive, par exemple, lorsque devant le spectacle de civilisations déchues, on demande l'explication de ces décadences et de ces ruines à des changements de climats. Sûrement, il y a eu de tels changements depuis l'époque quaternaire ; mais peut-on appliquer leurs effets à l'histoire humaine ? On reste inquiet devant de telles hypothèses, dont le moindre défaut n'est pas de détourner la question et de fermer la porte à des recherches qui prenant l'histoire pour base, n'auraient sans doute pas dit leur dernier mot. Il est temps de conclure. Nous avons connu longtemps la géographie incertaine de son objet et de ses méthodes, oscillant entre la géologie et l'histoire. Ces temps sont passés. Ce que la géographie, en échange du secours qu'elle reçoit des autres sciences, peut apporter au trésor commun, c'est l'aptitude à ne pas morceler ce que la nature rassemble, à comprendre la correspondance et la corrélation des faits, soit dans le milieu terrestre qui les enveloppe tous, soit dans les milieux régionaux où ils se localisent. Il y a là, sans nul doute, un bénéfice intellectuel qui peut s'étendre à toutes les applications de l'esprit. En retraçant les voies par lesquelles la géographie est arrivée à éclairer son but et à affermir ses méthodes, on reconnaît qu'elle a été guidée par le désir d'observer de plus en plus directement, de plus en plus attentivement, les réalités naturelles. Cette méthode a porté ses fruits ; l'essentiel est de s'y tenir.

Paul Vidal De La Blache. 